\title{
BMJ Open Protocol for integrating mental health services into primary healthcare facilities: a qualitative study of the perspectives of patients, family members and healthcare providers in rural Bangladesh
}

\author{
Aliya Naheed (D) ," Mir Nabila Ashraf (D) , ${ }^{1}$ Nantu Chakma, ${ }^{1}$ \\ Hannah Maria Jennings (D) , 2,3,4 Papreen Nahar (i) ${ }^{5}$
}

To cite: Naheed A, Ashraf MN, Chakma N, et al. Protocol for integrating mental health services into primary healthcare facilities: a qualitative study of the perspectives of patients, family members and healthcare providers in rural Bangladesh. BMJ Open 2022;12:e052464. doi:10.1136/ bmjopen-2021-052464

- Prepublication history and additional supplemental material for this paper are available online. To view these files, please visit the journal online (http://dx.doi.org/10.1136/ bmjopen-2021-052464)

MNA and NC contributed equally.

Received 16 April 2021

Accepted 04 January 2022

Check for updates

(c) Author(s) (or their employer(s)) 2022. Re-use permitted under CC BY-NC. No commercial re-use. See rights and permissions. Published by BMJ.

For numbered affiliations see end of article.

Correspondence to

Dr Aliya Naheed;

anaheed@icddrb.org

\section{ABSTRACT}

Introduction In Bangladesh, Non-communicable diseases (NCDs) account for $67 \%$ of all deaths. Mental health services are not available in routine healthcare at the primary facilities in Bangladesh. The protocol is for a qualitative study that seeks to understand the perceptions, beliefs and norms regarding common mental disorders (CMDs) among patients with NCD with and without CMDs to identify barriers to accessing mental health services in rural communities in Bangladesh. We also aim to explore the feasibility of integrating mental healthcare into routine NCD services at primary health facilities in rural Bangladesh.

Methods and analysis This study will be conducted at the outpatient departments in two subdistrict hospitals and one district hospital in Munshiganj district in Bangladesh. We will purposefully select patients with hypertension and diabetes from the patient inventory generated from a recently completed randomised control trial titled 'Control of Blood Pressure and Risk Attenuation Bangladesh, Pakistan, Sri Lanka' in two subdistricts in Munshiganj district in Bangladesh. The selected participants will be screened for CMD using the Depression, Anxiety and Stress Scale - 21 Items (DASS-21) over the telephone. Sixty in-depth interviews with patients and family members, 8-10 key informant interviews with healthcare providers and 2 focus group discussions with community health workers will be held following consent.

The study is conceptualised under Levesque et a/s framework. Thematic analysis will be applied following the study objectives and key issues, and commonly emerging topics generated by the data. The findings will be presented anonymously to corroborate the interpretation. Ethics and dissemination Approval has been obtained from the Institutional Review Board at icddr,b (PR-19108) and the University of York (HSRGC/2020/382/F). Written informed consent or audio recording consent form in Bangla will be obtained. For dissemination, we will invite representatives of the collaborating institutions to share the findings in national or international conferences and peer-reviewed journals.
Strengths and limitations of this study

We have purposefully selected facilities where Noncommunicable diseases (NCD) strengthening has already taken place, which may not entirely represent a regular health facility without NCD strengthening.

- Rapport building is difficult while conducting qualitative interviews by phone, which might compromise the qualitative data.

- We have selected patients of the previous study who are already sensitised, which may positively portray patients' perspectives in the future analyses.

- We have included family members who play a vital role in patient's decision-making and generally get ignored.

- The telephone interview has created a new entity of data collection in an emergency situation, such as COVID-19.

\section{INTRODUCTION}

Non-communicable diseases (NCDs) such as cardiovascular disease (CVD), diabetes, cancer and respiratory diseases account for approximately $71 \%$ of deaths globally. ${ }^{1}$ In low -and middle-income countries (LMICs), NCDs account for the death of nearly 32 million people each year. ${ }^{1} \mathrm{~A}$ significant association between CVD and common mental disorders (CMDs) such as depression and anxiety has been observed in several epidemiological studies. ${ }^{2-4}$ Evidence suggests that $9 \%-23 \%$ of people with one or more chronic physical conditions (such as cancer, diabetes and CVD) suffer from a comorbidity with depression. ${ }^{5}$

According to the national reports gathered by WHO's South East Asia regional office, of the total deaths in South Asia, the proportion 
attributable to selected NCDs ranges from $7 \%$ in Nepal to $40 \%$ in the Maldives. ${ }^{6}$ CVD can be attributed to $34 \%$ of deaths in Sri Lanka, 30\% in Bangladesh, 29\% in Pakistan and $27 \%$ in India. ${ }^{7-10}$ In South Asia, patients with diabetes and depression are more likely to have a poor quality of life, increased medical complications and higher mortality compared with patients with diabetes without depression. ${ }^{112}$ Patients with diabetes and depression are more prone to have cardiac events and complications, such as diabetic retinopathy, increased risk of disability and increased health service costs compared with patients with diabetes without depression. ${ }^{13-15}$

NCDs account for $67 \%$ of all deaths in Bangladesh, according to the WHO. ${ }^{16}$ The most common NCDs include CVDs, diabetes mellitus, cancer and chronic respiratory diseases. ${ }^{17} \mathrm{~A}$ meta-analysis showed that the pooled prevalence of diabetes is $7.8 \%$ (95\% CI: 6.4-9.3) which is consistent with many other LMICs countries such as Sri Lanka (8.0\%), India (8.6\%) and China $(9.6 \%) .{ }^{18-21}$ The prevalence of the CVD is $5 \%$ which is higher in urban areas $(8 \%)$ compare to rural areas $(2 \%) .{ }^{22}$ Although there is a dearth evidence of the magnitude of CMDs in NCDs in Bangladesh, the prevalence of mental disorders has been reported to vary from $6.5 \%$ to $31.0 \%$ among adults and from $13.4 \%$ to $22.9 \%$ among children. ${ }^{23}$ There has been limited evidence from hospital-based studies demonstrating the prevalence of depression to be $45.2 \%$ among patients with diabetes and $70 \%$ among patients who had a post stroke. ${ }^{24}$

The WHO highly recommends integrating mental health services into primary health. ${ }^{25}$ In Bangladesh, there is a very low level of awareness of mental health at the community level. The stigma around mental health prevents patients from seeking mental health services when visiting primary healthcare facilities. ${ }^{26}{ }^{27}$ Healthcare providers in primary healthcare facilities often fail to treat patients suffering from mental health issues and are unlikely to refer patients to specialised mental health facilities and departments. ${ }^{28} 29$

\section{Rationale and objectives}

The government plans to strengthen NCD corners at subdistrict hospitals also known as Upazila Health Complex (UHC), as outlined in the operational plan for NCDs in Bangladesh. However, there are no plans or strategies to integrate mental healthcare into NCD care at government primary healthcare facilities. ${ }^{30} \mathrm{~A}$ sister project of the current study titled 'Behavioural Activation for Comorbid Depression in Non-communicable Disease (BEACON)' reported about the lack of preparedness of NCD centres for managing the treatment of CMDs such as depression and the absence of guidelines, proper recording systems and training. ${ }^{31}$

Family members often play a critical role in the care of patients and have a different perspective to that of the patient. ${ }^{32}$ The family members of mentally ill patients are often socially isolated due to their relatives' condition. ${ }^{33}$ Women are more prone to suffer from NCDs such as hypertension compared with men. ${ }^{34}$ To mobilise any mental health programme, it is crucial to understand the perceptions and challenges of family members in addition to patients, especially for the women for whom their family members play a critical role in primary decisionmaking about healthcare. ${ }^{35}$ The perceptions of healthcare providers and identifying the challenges from their personal experiences should contribute to the design of any intervention for patients and communities in the healthcare setting. ${ }^{36}$

We propose exploring the perspectives and experiences of patients with NCDs and their family members regarding accessing healthcare for CMDs in primary healthcare facilities, and exploring the perspectives of healthcare providers on barriers, facilitators and demand for mental healthcare in primary healthcare settings. The study will contribute to understanding the experiences of CMDs among the patients with NCD and their family members, and identify possible strategies to integrate mental health into primary healthcare in Bangladesh.

The specific objectives of the study are:

1. To understand the perceptions, beliefs and norms regarding CMDs among patients with NCDs and their close family members in order to identify barriers for accessing mental health service.

2. To assess the acceptability and feasibility of implementing mental health services at a primary health facility from a patients' perspective.

3. To explore the views of healthcare providers regarding the need of mental health services and the feasibility of integrating mental health services into primary healthcare.

\section{METHODS AND ANALYSIS \\ Study design \\ Qualitative.}

\section{Conceptual framework}

The study is conceptualised under the Levesque et $a l$ s framework ${ }^{37}$ for exploring patient-centred healthcare access and care pathways of CMD among patients with NCD in primary healthcare in rural Bangladesh (figure 1). This framework covers five dimensions of access to healthcare. They are: (1) approachability; (2)

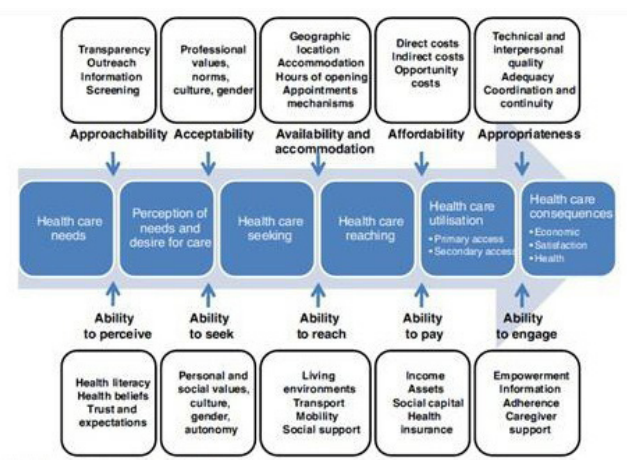

Figure 1 Levesque et al's framework. 
Table 1 Operational definition of dimension of the Levesque et al's framework

\begin{tabular}{|c|c|}
\hline Dimension & Definition \\
\hline Approachability & $\begin{array}{l}\text { Relates to the fact that people facing health needs can actually identify that some form of services exist, can be reached, } \\
\text { and have an impact on the health of the individual. In addition, approachability of services, the notion of ability to perceive } \\
\text { need for care among populations is important and determined by the factors like- health literacy, knowledge about health } \\
\text { and beliefs related to health and sickness. }\end{array}$ \\
\hline Acceptability & $\begin{array}{l}\text { Speaks about social and cultural features determining the opportunities for individuals to recognise the aspects of the } \\
\text { service and the appropriateness for the persons to seek care. }\end{array}$ \\
\hline Affordability & $\begin{array}{l}\text { It is the economic capacity for people to spend resources and time to use suitable services. It results from direct prices of } \\
\text { services and related expenses in addition to opportunity costs related to loss of income. Therefore it can differ by type of } \\
\text { services and depends on the capacity to generate the resources to pay for care. }\end{array}$ \\
\hline Appropriateness & $\begin{array}{l}\text { Refers to fit between services and patients need, the amount of care spent in assessing health problems and determining } \\
\text { the correct treatment and the services provided. }\end{array}$ \\
\hline
\end{tabular}

acceptability; (3) availability and accommodation; (4) affordability; and (5) appropriateness. In addition to that we will also understand the barriers of the mental health services and recommendations to improve pathway to care (table 1). Levesque et al also identify the dimensions with corresponding abilities from patient perspective that include (1) ability to perceive (healthcare needs, perception of needs and desire for care); (2) ability to seek; (3) ability to reach; (4) ability to pay; and (5) ability to engage. $^{37}$

\section{Study sites}

We will conduct the study in Munshiganj district, a largely rural district in central Bangladesh with a population of 1.4 million. We have selected Munshiganj district as it has both secondary (district hospital) and primary healthcare facilities (Louhajang and Sirajdikhan UHCs) where

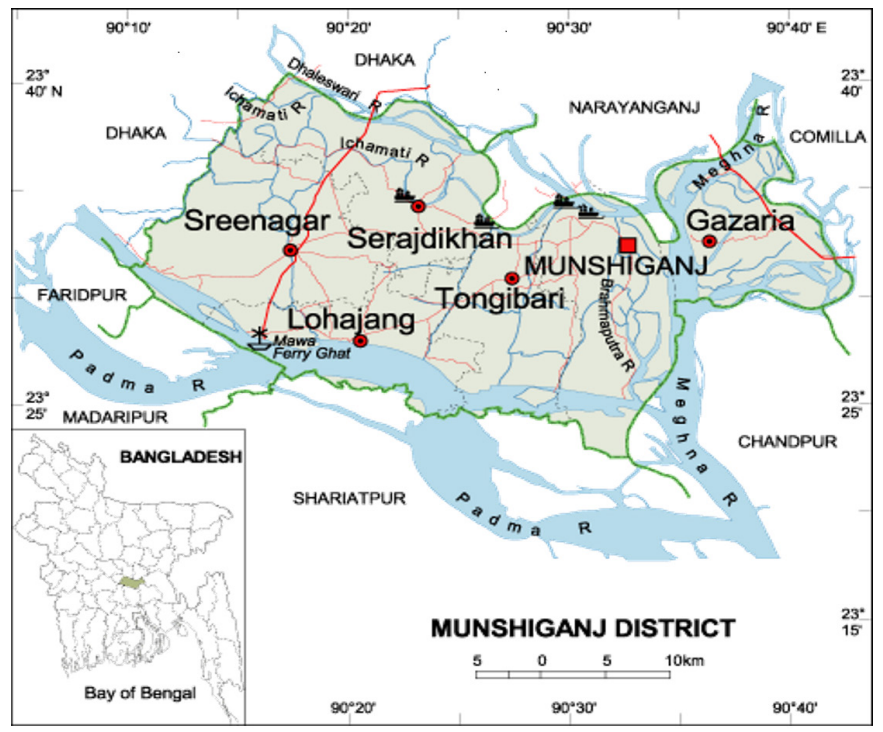

Source: Maps of Bangladesh

Figure 2 GPS location of Munshiganj district. we can conduct the research (figure 2). The reasons of selecting these facilities are: the NCD corners of these facilities have been strengthened by the NCD Control Program of the Directorate General of Health Services over the past few years; and the service providers are near to Dhaka; the community people are familiar and sensitised with the research group and the research activities due to a recent implementation of a multi component intervention trial titled 'Control of Blood Pressure and Risk Attenuation' (COBRA-BPS); and there is a pool of patients with NCD recruited under the recent COBRA-BPS study. ${ }^{389}$ Due to the COVID-19 situation, field movement is restricted and hence recruitment is challenging. Therefore, we will recruit participants from this patient pool to conduct interviews remotely (by phone).

\section{Study population}

We will purposefully select patients with hypertension and diabetes. For objectives 1 and 2, the primary target group will be adult men and women aged 30 years and above living with hypertension and diabetes, and their family members. For objective 3, the managers (civil surgeon of the District Hospital and Upazila Health \& Family Planning Officer of UHCs), medical officers, nurses and community health workers (CHWs) who are responsible for NCD care deployed at the selected healthcare facilities will be selected for key informant interviews (KIIs) (table 2).

Selection of patients and family members for in-depth interviews We will apply DASS 21 to screen for CMD among patients with NCD at each facility. We will recruit 10 patients from each of the three selected facilities ( $\mathrm{n}=30$ in total), which will include 5 with a CMD and 5 without a CMD per facility. All together we will conduct in-depth interviews (IDIs) with 30 patients, $50 \%$ of whom have a CMD (table 2). Additionally, an adult family member of each patient who is involved in their care and/or decision-making 
Table 2 Number of study participants for IDI, KII and FGDs across different providers

\begin{tabular}{|c|c|c|c|c|c|c|}
\hline \multirow[b]{2}{*}{ Study sites } & \multicolumn{4}{|c|}{ In-depth interviews (IDI) } & \multirow{2}{*}{$\begin{array}{l}\text { Key informant } \\
\text { interviews (KII) } \\
\text { Providers (civil } \\
\text { surgeon, UH\&FPO } \\
\text { MO, nurse) }\end{array}$} & \multirow{2}{*}{$\begin{array}{l}\text { Focus group } \\
\text { discussion } \\
\text { (FGD) with } \\
\text { CHWs(6-8 in } \\
\text { a group) }\end{array}$} \\
\hline & $\begin{array}{l}\text { Patients with } \\
\text { NCD with } \\
\text { CMD }\end{array}$ & $\begin{array}{l}\text { Family } \\
\text { members }\end{array}$ & $\begin{array}{l}\text { Patients with } \\
\text { NCD without } \\
\text { CMD }\end{array}$ & $\begin{array}{l}\text { Family } \\
\text { members }\end{array}$ & & \\
\hline Sirajdikhan subdistrict hospital & 5 & 5 & 5 & 5 & 3 & 1 \\
\hline Louhajang subdistrict hospital & 5 & 5 & 5 & 5 & 3 & 1 \\
\hline Total & 15 & 15 & 15 & 15 & 10 & 3 \\
\hline
\end{tabular}

CHW, community health worker; CMD, common mental disorder; MO, medical officer; NCD, Non-communicable disease; UH\&FPO, Upazila Health \& Family Planning Officer.

for health seeking of the patient will be recruited $(n=30$ total). We will invite the family members over the phone, and those who will provide voluntary consent will be interviewed at their convenience.

The proposed study has been designed considering the challenges of conducting field research during the COVID-19 pandemic. We will use already available records of patients diagnosed as having hypertension and/or other NCDs recruited from Sirajdikhan and Louhajang UHCs in Munshiganj district under a recently completed study titled 'COBRA-BPS' which has been led in Bangladesh by the same Principal Investigator (PI) from icddr,b and lead author of the paper (AN). ${ }^{40}$ The study record includes data of 167 individuals recruited from the two UHCs and 37 (22\%) participants had reported to have visited the respective facility at least once during the trial period between 2017 and 2019 for seeking care for hypertension; 20 (12\%) participants visited the respective facility for a health reason other than an NCD. For the District Hospital site, we will prospectively list the patients who would visit the outpatient department for NCD care and approach to them for assessment of DASS-21 following a written consent and recruit 10 patients (5 with CMD and 5 without CMD).

We will ask each recruited patient to provide the information of an adult family member who is involved in their care and may be interested in being involved in the study. The family member will be contacted by the research team and be given information about the study. Informed consent will be taken if they are interested in taking part before being recruited. If they decide not to take part in the study, the research team will discuss with the patient about recruiting another family member involved in their care.

\section{Selection of the healthcare providers}

KIIs will be conducted with the civil surgeon of Munshiganj district, director of the District Hospital and upazila health and family planning officers at the Sirajdikhan and Louhajang UHCs. In addition, we will select at least one medical officer and one nurse for KIIs at each of the selected facilities designated for NCD care. The study PI and other investigators will contact potential key informants over the phone and inform them about the study and request their support. If participants express an interest in participating information about the study will be shared with them and they will be provided with a scanned copy of the consent form over Viber or WhatsApp, email or FAX as deemed appropriate, and will be contacted at a later date for a potential interview as per their convenience. Following informed verbal consent the KII will be conducted over the phone. Multiple sessions will be scheduled for completing the KII if the full interview cannot be done in a single session. If a participant is not comfortable to talk over the phone, we will arrange a face-to-face interview at a convenient time and location.

\section{Focus group discussions with CHWs}

We will invite CHWs who provide primary healthcare services at the community level. We will invite 6-8 CHWs from each of the facility for a focus group discussion (FGD). They will be approached by the research team over the phone and be provided with information about the study and invited to join an FGD. A scanned copy of the consent form will be shared via viber, WhatsApp or any other appropriate digital platform such as zoom. If they are interested in participating in the FGD, a time and date will be set when most of the CHWs under a UHC can gather at a time and location convenient for them.

\section{Data collection}

\section{Patient and family members}

IDI with patients and family members will take place at a time and place (either face to face at home or a private room in the health facility or by phone) convenient to the participant. This will be arranged in negotiation between the researcher and the participants. If the interviews are face to face the researchers will follow icddr,b COVID-19 safeguarding policy (social distancing, surface cleaning, wearing masks, etc). The interviews will follow a prepared topic guide and be audio recorded. The length of the interviews is expected to be between 30 and 60 minutes.

\section{The interviews will cover the following areas}

To explore patient and family members' perception, we will assess the accessibility of ability to perceive the service; 
approachability of transparency of the service and belief regarding mental health; acceptability of ability to seek, perceived belief and norms about mental health service, perceived need of mental health service; affordability of ability to pay for the service; availability and accommodation of mobility and availability of transportation, occupational flexibility; barrier: possible barriers of the service (online supplemental Annex 1.1, 1.2).

\section{Healthcare providers}

KII will take place by phone, or if preferred face to face. If they take place face to face all COVID-19 protocols (social distancing, surface cleaning, wearing masks, etc) will be followed. The interviews will follow a topic-guide, be audio recorded and expected tobe between 45 and 60 minutes .

\section{The interviews will cover the following areas}

To understand the perspective of the healthcare providers, we will assess the accessibility of healthcare needs to seek healthcare services; approachability of information regarding available treatments and services and outreach activities; availability of existing services in the primary healthcare centre; affordability: cost of the existing service system, price of care and opportunistic cost, capacity to generate economic resources; availability and accommodation of appointment mechanism, hours of opening; barrier of the system for giving service to the patient and modifications that are required to improve the system; recommendations of the possible ways to integrate mental health in to primary healthcare and ways to mitigate potential risks (online supplemental Annex 1.3).

\section{Community health workers}

FGDs will take place following icddr,b COVID-19 safeguarding policies (surface cleaning social distancing, wearing masks, etc) at a time and place convenient for the participants, arranged by the researchers. The FGDs will be audio recorded and expected to be between 60 and 90 minutes.

\section{The FGDs will cover the following information}

To understand perspective of CHWs, we will assess accessibility of healthcare needs to seek healthcare services; approachability of information regarding available treatments and services and outreach activities; approachability of information regarding available treatments and services and outreach activities; availability of existing services in the primary healthcare centre; barrier of the system for giving service to the patient and modifications that are required to improve the system; recommendations of the possible ways to integrate mental health in to primary healthcare and ways to mitigate potential risks (online supplemental Annex 1.4).

\section{Data management}

Every participant will be assigned a unique identification number. Interviews and FGDs will be recorded digitally and verbatim responses will be transcribed line by line.
Participants may be contacted following the interviews and asked for additional information if adequate information is not obtained. Researchers involved in data collection will maintain regular field notes on themes and subthemes emerged during field data collection. Transcription will be conducted in Bengali and directly coded using a code list (English) developed by the bilingual researchers in the team. We will use NVivo (V.12) developed by QSR International for extracting data based on codes and sub codes under the emerging themes and subthemes. Data will be analysed in both inductive and deductive approaches.

\section{Data analysis}

A qualitative approach will be taken to conduct this study. All qualitative recorded data will be transcribed as soon as they are collected. A thematic analysis will be employed for data analysis. Thematic analysis will allow categorising, investigate and reflect on the data to examine in details and with a richness. ${ }^{41}$ The content of the data will be systematically categorised by identifying themes or patterns for smaller unites or codes. ${ }^{42}$ This coding process will employ both inductive and deductive coding, which will include codes either predetermined or to appear from the data. ${ }^{43}$ The analysis will be done using the "conceptual framework of access to health care' by Levesque $e t$ al by the research team members. One qualitative expert will ensure the quality assurance of the data coding. We will adhere to the Consolidated Criteria for Reporting Qualitative research guideline for the reporting. ${ }^{44}$

Levesque's framework conceptualises access to healthcare from both the demand and supply sides. It identifies various determinants that can have impacts on the patients' access to health services. The current study will explore access issues from the patients, caregivers (demand side) and providers (supply side), using the five determinants proposed by the framework mentioned in an earlier section. The coded data will be interpreted and embedded in a discussion on the five above mentioned access-related determinants and their interrelations. Thematic analysis will be done to answer the relevant key questions by analysing data and triangulation would be done to derive common themes emerging across the sites.

In order to understand the complexities of integrated care and data permit, we will explore further to elicit and critique a model(s) for integration from the KIIs and IDIs under the following themes: (1) what do patients/ providers think will work? (2) what will it take to deliver the model? (3) what potential risks must be mitigated? and (4) what recommendations can be pulled out on actors, settings, equipment/supplies, other health system considerations for the policy-makers? The research team members will be vigilant about the environment and take notes while conducting interviews in order to describe the setting during the interviews and the notes during the interviews will be taken in to account while analysing data in order to reduce subjectivity of interpretation of results. 


\section{Patient and public involvement}

Patients or the public were involved in the designing the study and preparing the guidelines. At the developmental phase of writing the protocol, we have had several discussions with the patients that have helped us developing the methodology. We have also tested the guidelines with patients and healthcare providers and modified them accordingly.

\section{Ethics and dissemination}

For patient recruitment, we will obtain consent at two stages; first before assessment of CMD applying DASS-21 over phone among the patients with NCD listed in the inventory to be created for identifying 10 patients from each facility ( 5 with CMD and 5 without CMD) and second before conducting IDI following assessment of CMD. During the consenting process of stage 1 recruitment, the interviewer will read the information sheet about the study, answer any questions and will read the consent form to the participants and answer any questions over the phone. This process will be audio recorded following the verbal consent of the participant. Those who would give permission to complete the DASS-21 for assessment of a CMD will be recruited into the study.

Each participant will be requested to participate in a subsequent face to face or phone interview along with a family member after 2 weeks of recruitment, if he/she is found eligible. Those met the criteria will be contacted over the phone after 2 weeks to conduct an interview following a voluntary consent. Any participants found likely having a CMD according to the DASS-21 assessment will be provided government authorised mental health support phone numbers. ${ }^{45}$ The adult family members (18 years or older) as suggested by the patient with NCD will be recruited following consent, unless $\mathrm{s} /$ he is not physically or mentally compromised.

The IDIs with the participants and their family members will be preferably conducted over phone, but we will also keep the provision for face-to-face interviews if the respondents are not comfortable to talk over phone or s/ he does not have a phone on his/her own. In addition, face-to-face interviews will be conducted with patients if the researchers are not confident that data obtained over phone are likely to provide valid information (eg, if the participant is very distracted or short of hearing). All phone calls will be made by the research staff and the cost of phone bills will be borne by the study. Any cost incurred by the participants or research staff due to use of phones will be reimbursed by the study.

KIIs with the health managers will be conducted via zoom or any other virtual platform or face to face, provided preferred by the key informants. ${ }^{4647}$ FGDs will be conducted via face-to-face session and the discussion will be audio recorded with the verbal consent of the participants however if situation worsen we will use the zoom for FGDs that has been a common platform specially amid this COVID-19 situation. ${ }^{47}$ In Bangladesh, now there has been no lockdown due to a reduced infection rate of COVID-19. ${ }^{48}$ The number of group members will be limited to 6-8 participants in each FGD.

We will assure the recommended social distancing between the participants during the FGD, IDI and KII by ensuring a sitting arrangement so that the participants are maintained at least at 3 feet distance from one another. Additionally, participants will be provided masks to wear during the face-to-face interviews and FGDs. Researchers will wear the recommended personal protective equipment during any face-to-face interactions.

Any participant who reports having a fever, cough or any other symptoms of COVID-19 will not be invited to participate in the face-to-face interview. We will refer participants with symptoms of COVID-19 to the nearest government UHC for COVID-19 testing and care.

We have received ethical approval from icddr,b (PR19108) on 13 January 2020 and from the University of York (HSRGC/2020/382/F) on 20 March 2020. Following the emerging of COVID-19 situation, we have done amendment of the protocol and received ethical approval from icddr,b (PR-19108) on 13 September 2020 and from the University of York (HSRGC/2020/382/F) on 20 October 2020. Information about the study will be provided in a participant information letter in Bangla (to be read verbally and given in writing to participants). A written informed consent or audio recording consent form in Bangla will be obtained and participants will be informed of their right to withdraw from the study. All the collected information will be kept in confidential and can be accessible only to the study team. If any verbatim is presented in the results it will be published anonymously. For dissemination, we will invite representatives of the collaborating institutions to share the findings in national and international conferences and peer reviewed journals.

\section{Progress untill April 2021}

The guidelines are pretested and revised accordingly. A list of participants has been generated from the list of participants included in the COBRA-BPS study that was conducted in the same study settings, and a patient inventory has been created. Due to the current COVID-19 situation, the study activities were delayed pending on a thorough training of the study team members on biosecurity and personal safety during the COVID-19 pandemic.

Between 14 September 2020 and 20 October 2021, we approached to 116 patients over the phone, and 40 participants responded positively. We screened CMD among 32 participants using DASS-21 scale (23 phone interviews and 9 face-to-face interviews). Among them, 12 participants were recruited from Louhajang Upazila Health Complex (9 CMD and 3 non-CMD), 11 participants from Shirajdikhan Upazila Health Complex (5 CMD and 6 non-CMD) and 9 participants from Munshiganj General Hospital (4 CMD and 5 non-CMD). We contacted the participants after 2 weeks of CMD screening and conducted IDIs following a voluntary consent. So far, we have completed IDIs with 20 participants and family members of 10 participants over phone; one 
key-informant interview was conducted over a face-to-face interview. We have completed data coding of interviewed patients and family members.

\section{Author affiliations}

${ }^{1}$ Initiative for Noncommunicable Diseases, International Centre for Diarrhoeal Disease Research Bangladesh, Dhaka, Bangladesh

${ }^{2}$ Department of Health Sciences, University of York, York, UK

${ }^{3}$ Hull York Medical School, York, UK

${ }^{4}$ Institute for Global Health, UCL, London, UK

${ }^{5}$ Department of Global Health and Infection, Brighton and Sussex Medical School, University of Sussex, Brighton, UK

Acknowledgements This review received support and contributions from many of our colleagues at icddr,b and IMPACT NIHR Global Health Research Group. icddr,b acknowledges with gratitude the commitment of National Institute for Health Research to its research efforts. icddr,b is also grateful to the Governments of Bangladesh, Canada, Sweden and the UK for providing core/unrestricted support.

Contributors AN conceived the study and the study design and drafted the protocol. PN, HMJ, MNA and NC contributed to finalising the design. All authors contributed to writing the manuscript.

Funding This work was partly funded by the UK National Institute for Health Research (NIHR) Global Health Research as part of the IMPACT project (Project Number 17/63/130). This research was commissioned by the NIHR using Official Development Assistance (ODA) funding.

Map disclaimer The inclusion of any map (including the depiction of any boundaries therein), or of any geographic or locational reference, does not imply the expression of any opinion whatsoever on the part of BMJ concerning the legal status of any country, territory, jurisdiction or area or of its authorities. Any such expression remains solely that of the relevant source and is not endorsed by BMJ. Maps are provided without any warranty of any kind, either express or implied.

Competing interests None declared.

Patient consent for publication Not applicable.

Provenance and peer review Not commissioned; externally peer reviewed.

Supplemental material This content has been supplied by the author(s). It has not been vetted by BMJ Publishing Group Limited (BMJ) and may not have been peer-reviewed. Any opinions or recommendations discussed are solely those of the author(s) and are not endorsed by BMJ. BMJ disclaims all liability and responsibility arising from any reliance placed on the content. Where the content includes any translated material, BMJ does not warrant the accuracy and reliability of the translations (including but not limited to local regulations, clinical guidelines, terminology, drug names and drug dosages), and is not responsible for any error and/or omissions arising from translation and adaptation or otherwise.

Open access This is an open access article distributed in accordance with the Creative Commons Attribution Non Commercial (CC BY-NC 4.0) license, which permits others to distribute, remix, adapt, build upon this work non-commercially, and license their derivative works on different terms, provided the original work is properly cited, appropriate credit is given, any changes made indicated, and the use is non-commercial. See: http://creativecommons.org/licenses/by-nc/4.0/.

\section{ORCID iDs}

Aliya Naheed http://orcid.org/0000-0002-6016-5603

Mir Nabila Ashraf http://orcid.org/0000-0002-8908-3273

Hannah Maria Jennings http://orcid.org/0000-0002-8580-0327

Papreen Nahar http://orcid.org/0000-0002-5817-8093

\section{REFERENCES}

1 Noncommunicable diseases, 2018. Available: https://www.who.int/ news-room/fact-sheets/detail/noncommunicable-diseases

2 Rajan S, McKee M, Rangarajan S, et al. Association of symptoms of depression with cardiovascular disease and mortality in low-, middleand high-income countries. JAMA Psychiatry 2020;77:1052-63.

3 Cohen BE, Edmondson D, Kronish IM. State of the art review. depression, stress, anxiety, and cardiovascular disease. Am J Hypertens 2015;28:1295-302.
4 Batelaan NM, Seldenrijk A, Bot M, et al. Anxiety and new onset of cardiovascular disease: critical review and meta-analysis. $\mathrm{Br} J$ Psychiatry 2016;208:223-31.

5 Scott KM, Bruffaerts R, Tsang A, et al. Depression-anxiety relationships with chronic physical conditions: results from the world mental health surveys. J Affect Disord 2007;103:113-20.

6 Ghaffar A, Reddy KS, Singhi M. Burden of non-communicable diseases in South Asia. BMJ 2004;328:807-10.

7 WHO. Risk of premature death due to NCDS Bangladesh. Bangladesh: WHO, 2016.

8 WHO. Risk of premature death due to NCDS India. India: WHO, 2016.

9 WHO. Risk of premature death due to NCDS Sri Lanka. Sri Lanka: WHO, 2016.

10 WHO. Risk of premature death due to NCDS Pakistan. Pakistan: WHO, 2016.

11 Safita N, Islam SMS, Chow CK, et al. The impact of type 2 diabetes on health related quality of life in Bangladesh: results from a matched study comparing treated cases with non-diabetic controls. Health Qual Life Outcomes 2016;14:129.

12 Rezia R, Islam A, Islam SMS. Depressive symptoms among participants with type 2 diabetes in Southeast Asia: a systematic review. Journal of Diabetology 2018:9:19.

13 Pradeepa R, Mohan V. Prevalence of type 2 diabetes and its complications in India and economic costs to the nation. Eur J Clin Nutr 2017:71:816-24.

14 Zuberi SI, Syed EU, Bhatti JA. Association of depression with treatment outcomes in type 2 diabetes mellitus: a cross-sectional study from Karachi, Pakistan. BMC Psychiatry 2011;11:1-6.

15 Bajaj S, Agarwal SK, Varma A, et al. Association of depression and its relation with complications in newly diagnosed type 2 diabetes. Indian J Endocrinol Metab 2012;16:759.

16 World Health Organization. Noncommunicable diseases (Ncd) country profiles, Bangladesh, 2018. Available: https://www.who.int/ $\mathrm{nmh} /$ countries/bgd_en.pdf?ua $=1$

17 Biswas T, Pervin S, Tanim MIA, et al. Bangladesh policy on prevention and control of non-communicable diseases: a policy analysis. BMC Public Health 2017;17:1-11.

18 Katulanda P, Constantine GR, Mahesh JG, et al. Prevalence and projections of diabetes and pre-diabetes in adults in Sri Lanka-Sri Lanka Diabetes, Cardiovascular Study (SLDCS). Diabet Med 2008;25:1062-9.

19 Anjana RM, Pradeepa R, Deepa M, et al. Prevalence of diabetes and prediabetes (impaired fasting glucose and/or impaired glucose tolerance) in urban and rural India: phase I results of the Indian Council of medical Research-INdia diabetes (ICMR-INDIAB) study. Diabetologia 2011;54:3022-7.

20 Chan JCN, Malik V, Jia W, et al. Diabetes in Asia: epidemiology, risk factors, and pathophysiology. JAMA 2009;301:2129-40.

21 Akhtar S, Nasir JA, Sarwar A, et al. Prevalence of diabetes and prediabetes in Bangladesh: a systematic review and meta-analysis. BMJ Open 2020;10:e036086.

22 Chowdhury MZI, Haque MA, Farhana Z, et al. Prevalence of cardiovascular disease among Bangladeshi adult population: a systematic review and meta-analysis of the studies. Vasc Health Risk Manag 2018;14:165-81.

23 Hossain A, Rehena J, Razia MS. Mental health disorders status in Bangladesh: a systematic review. JOJNHC 2018;7:1-4.

24 Islam AKMM, Majumder AAS. Hypertension in Bangladesh: a review. Indian Heart J 2012;64:319-23.

25 World Health Organization. Integrating mental health services into primary health care, 2007. Available: http://www. who. int/mental health/policy/services/en/index. html

26 Henderson C, Evans-Lacko S, Thornicroft G. Help seeking, and public health programs. Am J Public Health 2013;103:777-80.

27 World Health Organization. WHO-AIMS report on mental health system in Bangladesh. WHO, 2007.

28 Hossain MD, Ahmed HU, Chowdhury WA, et al. Mental disorders in Bangladesh: a systematic review. BMC Psychiatry 2014;14:216.

29 Bangladesh, G.o. National Institute of mental health and hospital, 2020. Available: https://nimhbd.com/

30 Directorate General of Health Services. 4th Health, Population and Nutrition Sector Programme. Operational Plan. Non Communicable Disease Control. Dhaka: Ministry of Health and Family Welfare, Bangladesh, 2017. http://www.dghs.gov.bd/images/docs/OP/2018/ NCDC.pdf

31 Wright J, Mazumdar P, Barua D, et al. Integrating depression care within ncd provision in Bangladesh and Pakistan: a qualitative study. Int J Ment Health Syst 2020;14:1-13.

32 Ahmad FS, Barg FK, Bowles KH, et al. Comparing perspectives of patients, caregivers, and clinicians on heart failure management. $J$ Card Fail 2016;22:210-7. 
33 Lefley HP. Family burden and family stigma in major mental illness. Am Psychol 1989;44:556-60.

34 Chowdhury MZI, Rahman M, Akter T, et al. Hypertension prevalence and its trend in Bangladesh: evidence from a systematic review and meta-analysis. Clin Hypertens 2020;26:1-19.

35 Roy PK, Haque S, Jannat A, et al. Contribution of women to household income and decision making in some selected areas of Mymensingh in Bangladesh. Progressive Agriculture 2017;28:120-9.

36 Weiss JM, Pickhardt PJ, Schumacher JR, et al. Primary care provider perceptions of colorectal cancer screening barriers: implications for designing quality improvement interventions. Gastroenterol Res Pract 2017;2017:1-9.

37 Levesque J-F, Harris MF, Russell G. Patient-Centred access to health care: conceptualising access at the interface of health systems and populations. Int J Equity Health 2013:12:18.

38 Jafar TH, Gandhi M, de Silva HA, et al. A community-based intervention for managing hypertension in rural South Asia. N Engl $J$ Med 2020;382:717-26.

39 Jafar TH, Jehan I, de Silva HA, et al. Multicomponent intervention versus usual care for management of hypertension in rural

Bangladesh, Pakistan and Sri Lanka: study protocol for a cluster randomized controlled trial. Trials 2017;18:1-12.

40 Naheed A, Haldane V, Jafar TH, et al. Patient pathways and perceptions of hypertension treatment, management, and control in rural Bangladesh: a qualitative study. Patient Prefer Adherence 2018;12:1437-49.

41 Braun V, Clarke V. Using thematic analysis in psychology. Qual Res Psychol 2006;3:77-101.

42 Erlingsson C, Brysiewicz P. A hands-on guide to doing content analysis. Afr J Emerg Med 2017;7:93-9.

43 Bernard HR. Research methods in anthropology: qualitative and quantitative approaches. Rowman \& Littlefield, 2017.

44 Tong A, Sainsbury P, Craig J. Consolidated criteria for reporting qualitative research (COREQ): a 32-item checklist for interviews and focus groups. Int J Qual Health Care 2007;19:349-57.

45 Zarrin F. Coping with autism in the age of quarantine. Dhaka Tribune, 2020.

46 Reñosa MDC, Mwamba C, Meghani A, et al. Selfie consents, remote rapport, and Zoom debriefings: collecting qualitative data amid a pandemic in four resource-constrained settings. BMJ Glob Health 2021;6:e004193.

47 Su I-A, Ceci S. "Zoom Developmentalists": Home-Based Videoconferencing Developmental Research during COVID-19 2021.

48 Shammi M, Bodrud-Doza M, Islam ARMT, et al. Strategic assessment of COVID-19 pandemic in Bangladesh: comparative lockdown scenario analysis, public perception, and management for sustainability. Environ Dev Sustain 2020:1-44. 